\title{
An Estimating Method for IT Project Expected Duration Oriented to GERT
}

\author{
Li Yu and Meiyun Zuo \\ School of Information, Renmin University of China, Beijing 100872, P.R. China \\ buaayuli@ruc.edu.cn zuomeiyun@263.net
}

\begin{abstract}
Reworking in developing software exists widely in IT project management. But the current estimating methods on project duration cannot deal well with this problem. In this paper, some key concepts, such as adjusted activity, adjusted GERT network, expected project duration, expected critical path, are firstly defined. Then authors study estimating method on project duration for different rework modes, including serial, paratactic, and combined structure by encapsulating of activities. Finally, a general estimating method on IT project duration oriented to GERT is presented, and detailed steps are illustrated with an example project on IT software development.
\end{abstract}

Keywords: Project Management, Project Schedule Estimation, Expected Duration

\section{INTRODUCTION}

In project management, project schedule often faces with the problem how to know project duration under the restriction of resource. PERT (Program Evaluation and Review Technique) and CPM (Critical Path Method) are the most important project management techniques [1], which have been created out in the need of Western industrial and military establishments to plan, schedule and control complex projects [2].

GERT (Graphical Evaluation and Review Technique) is another important analysis technique, being widely using in engineering management and enterprise producing for strong ability to describe reworking in project management [1].

Reworking exists in IT project management widely, and GERT can describe it well. But current method to estimate project duration only can deal with determinate project network without reworking. So, the problem how to estimate project duration with reworking became key technique for the using of GERT. Currently, few such researches are made in the world. Very few research is made on estimating project duration oriented to GERT by simulating method [3]. But it is difficult to compute accurately project duration, especially when times of simulating is few, the error is more obvious. The paper tries to resolve the problem by encapsulating of activities.

In next section, key concepts, such as expected duration and expected critical path etc, are presented. In section 3, author research on estimating method on IT project duration under all kinds of reworking cases, including serial, paratactic, and combined

Please use the following fornat when citing this chopter:

Yu, L., Zuo, M., 2007, in IFIP International Federation for Information Processing, Volume 255, Research and Practical Issues of Enterprise Information Systems II Volume 2, eds. L. Xu, Tjoa A., Chaudhry S. (Boston: Springer), pp. 15571566 . 
structure. General resolving method is given in section 4 . In section 5 , estimating method is used in a project example about the development of information system project.

\section{EXPECTED CRITICAL PATH AND EXPECTED PROJECT DURATION}

Under the condition with reworking, critical path can not be determined because the number of reworking for certain activity is not determinate. So there may be a lot of critical paths which have same activities in a project network diagram with reworking. Since the number of activities consisting of a critical path is determinate and each activity has determinate duration, project network diagram is determinate. Project duration could be infinite when reworking of certain activity always happened. If no reworking happened, GERT network became determinate network and project duration is determinate. Project duration can be computed by CPM and PERT methods. But both of the methods are not adapt to GERT network with reworking.

Although critical path and duration of the project based on GERT are not determinate, expect duration of each activity could be computed according to the probability distribution of the reworking. Then, according to expected duration of each activity, critical path of project can be computed by using CPM/PERT methods. Because this kind of critical path is based on expected duration of each activity, we call it as expected critical path of project, and corresponding duration as expected project duration. They are very useful in project schedule management when reworking could happen.

\section{ESTIMATING OF EXPECTED PROJECT DURATION FOE DIFFERENT REWORKING MODES}

\subsection{Including One Reworking Activity}

Here, being supposing that a project only includes one reworking activity $\mathrm{A}$, and its duration is a, the probability of reworking is p. As shown in Fig.1. Now, how to compute project duration?

Noting the expected project duration as $\mathrm{D}$, so

$$
D=a+(p * D)
$$

And

$$
D=\frac{a}{1-p}
$$




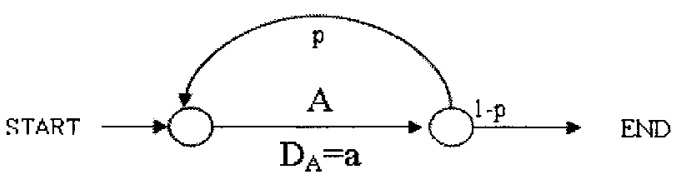

Figure 1. Project Including One Reworking Activity

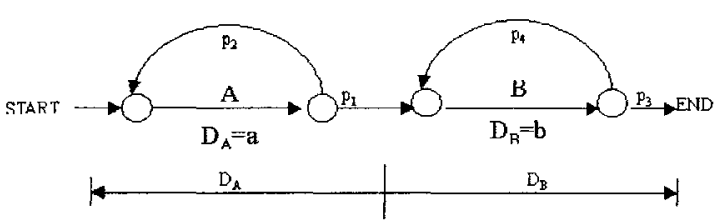

Figure 2. Serial Reworking

In the following, two extremities be analyzed,

(1) $p=0$

In this case, it means that no reworking exists, and expected project duration can be computed as following,

$$
D=\left.\frac{a}{1-p}\right|_{p=0}=a
$$

(2) $p=1$

In this case, it means that reworking always exists. As be showed in Equation (4), expected project duration is $+\infty$,

$$
D=\left.\frac{a}{1-p}\right|_{p=1}=+\infty
$$

Generally, bigger $\mathbf{p}$ shows higher reworking probability, so expected project duration is longer and $\mathrm{D}=\mathrm{D}(\mathrm{p})$ increased with increasing of reworking probability $p$.

Sometimes, it is usual that project allows top times of reworking. That is to say, after top number of reworking, the activity must be finished. The mode exists widely in project management practice. In this mode, computing expected project duration has practical meaning. Let's noting $\mathrm{Dn}$ as project duration while allowing $\mathrm{n}$ times of reworking for an activity, then the number list $\{\mathrm{Dn}\}(\mathrm{n}=1,2 \ldots)$ has following equations,

$$
\begin{aligned}
& D_{0}=a \quad D_{1}=a+p a \\
& D_{2}=a+p a+p^{2} a \ldots . \\
& D_{n}=a+p a+\ldots+p^{n} a \\
& \text { So, } \\
& D_{n}=\frac{a\left(1-p^{n+1}\right)}{1-p}
\end{aligned}
$$

Especially, while $n \rightarrow+\infty$, it means there is no limit for times of reworking. So 


$$
D=\lim _{n \rightarrow+\infty} D_{n}=\lim _{n \rightarrow+\infty} \frac{a\left(1-p^{n+1}\right)}{1-p}=\frac{a}{1-p}
$$

This conclusion is consistent with above the result.

\subsection{Including Lots of Reworking Activities}

\subsubsection{Serial Reworking}

As shown in Fig.2, a project includes two serial reworking activities A and B. Then

$$
D=D_{A}+D_{B}
$$

And DA and DB can be computed as Equation (1) and Equation (2),

$$
\begin{aligned}
& D_{A}=a+\left(p_{2} * D_{A}\right) \Rightarrow D_{A}=\frac{a}{1-p_{2}} \\
& D_{B}=b+\left(p_{4} * D_{B}\right) \Rightarrow D_{B}=\frac{b}{1-p_{4}}
\end{aligned}
$$

So

$$
D=D_{A}+D_{B}=\frac{a}{1-p_{2}}+\frac{b}{1-p_{4}}
$$

\subsubsection{Paratactic Reworking}

As shown in Fig.3, a project includes two paratactic reworking activities A and B. Then

$$
D=\max \left\{D_{A}, D_{B}\right\}
$$

And DA and DB can be computed as Equation (1) and Equation (2) , so

$$
\begin{aligned}
& D_{A}=a+\left(p_{2} * D_{A}\right) \Rightarrow D_{A}=\frac{a}{1-p_{2}} \\
& D_{B}=b+\left(p_{4} * D_{B}\right) \Rightarrow D_{B}=\frac{b}{1-p_{4}}
\end{aligned}
$$

Then

$$
D=\max \left\{D_{A}, D_{B}\right\}=\max \left\{\frac{a}{1-p_{2}}, \frac{b}{1-p_{4}}\right\}
$$




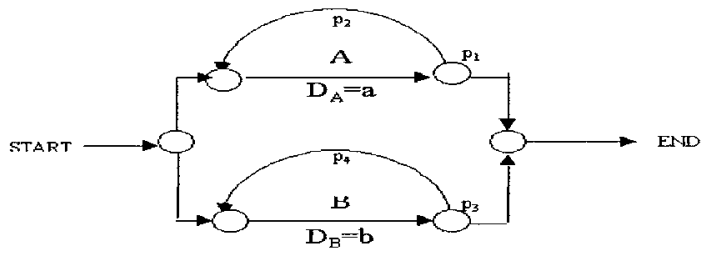

Figure 3. Paratactic Reworking

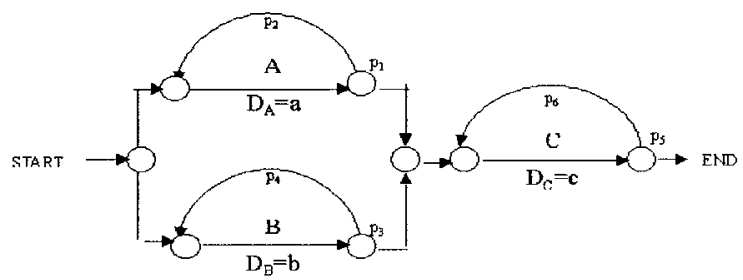

Figure 4. Combined Reworking

\subsubsection{Combined Reworking}

As shown in Fig.4, project includes serial reworking and paratactic reworking. Among of them, activities $\mathrm{A}$ and $\mathrm{B}$ are paratactic, being serial with activity $\mathrm{C}$. Then, expected project duration is

$$
D=\max \left\{D_{A}, D_{B}\right\}+D_{C}
$$

According to Equation (6) and Equation (7), D be computed as following

$$
\left.\left.D=\max \mathcal{D}_{A}, D_{B}\right\}+D_{C}=\max \frac{a}{1-p_{2}}, \frac{b}{1-p_{4}}\right\}+\frac{c}{1-p_{6}}
$$

\subsection{Reworking on Lots of Activities}

As shown in Fig.5, if activity B needs reworking, activity A also must rework. Reworking relates to two activities.

According to Equation (1), then

$$
D=(a+b)+\left(p_{2} * D\right)
$$

So

$$
D=\frac{a+b}{1-p_{2}}
$$




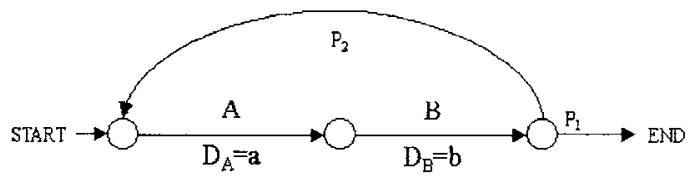

Figure 5. Reworking on Lots of Activities

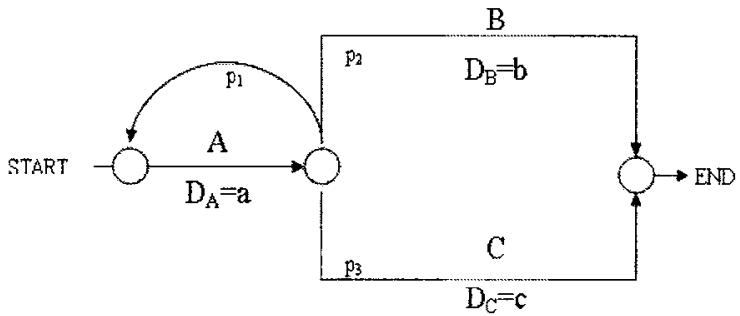

Figure 6. Multi-choices of Reworking

\subsection{Multi-choices of Reworking}

In IT project management, there are often lots of path choices for one activity. As shown in Fig.6, after activity $\mathrm{A}$ is finished, reworking could be happened at once or activity $B$ or $C$ could be executed. That is to say, after activity $A$ be finished, there are three path choices. In this case how to compute project duration?

In fact, this case can be transformed to two reworking choices mode. Activity B and $\mathrm{C}$ can be encapsulated to one virtual activity D, as be shown in Fig.7.

After encapsulating activity $\mathrm{B}$ and $\mathrm{C}$, according to the probability theory, the duration of virtual activity $\mathrm{D}$ can be computed as following

$$
D_{D}=\frac{p_{2} * b+p_{3} * c}{p_{2}+p_{3}}
$$

So, project duration is

$$
D=D_{A}+D_{D}=\frac{a}{1-p_{1}}+\frac{p_{2}{ }^{*} b+p_{3}{ }^{*} c}{p_{2}+p_{3}}=\frac{a+p_{2}^{*} b+p_{3}^{*} c}{p_{2}+p_{3}}
$$

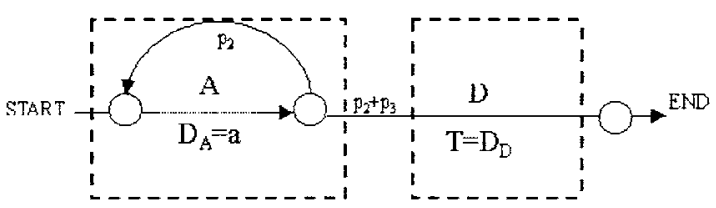

Figure 7. After Encapsulating Activity $B$ and C 


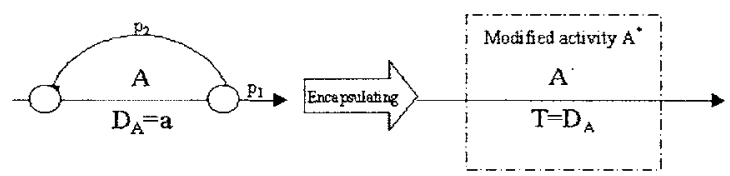

Figure 8. Encapsulating Activity

\section{ESTIMATING STEPS OF EXPECTED PROJECT DURATION ORIENTED GERT}

After discussing the computing method on expected project duration under different modes, we provide the general estimating steps of expected project duration oriented to GERT in the following.

\subsection{Encapsulating Reworking Activities}

The key step in estimating of expected project duration oriented to GERT is to transform GERT network with reworking activities to traditional determinate network without reworking activities. So the first step is to modify GERT network by encapsulating reworking activities, as shown in Figure 8. Activity A with reworking is transformed to modified activity $A^{*}$ without reworking by encapsulating. We call activity $A^{*}$ as adjusted activity of the activity $A$.

4.2 Computing of Duration of Adjusted Activity-- $D_{A}^{*}$

According to Equation (2), duration of adjusted activity $A^{*}$ is

$$
D_{A}^{*}=\frac{a}{1-p_{2}}
$$

We call it as adjusted duration of activity $\mathrm{A}$.

\subsection{Generating Adjusted GERT Network}

Doing as above two steps for all activities with reworking, we can generate new GERT network without reworking. We call the network as adjusted network diagram.

\subsection{Computing of Expected Critical Path and Expected Duration}

After eliminating reworking activity by encapsulating and generating adjusted GERT network, expected critical path and expected project duration can be computed by traditional CPM/PERT methods. 


\section{EXAMPLE}

For better explaining the above method, here we give a IT development project consisting of system design, buying hardware, software analysis, buying software, hardware examining, hardware setup, etc, as shown in Fig.9. Among these activities, two activities, hardware examining and system examining, could exist reworking. If there exists questions in hardware examining activity, hardware modifying activity happened. System examining happens according to trouble degree. All activities duration and reworking probability are shown in Fig.9. Now, we try to compute expected critical path and project duration.

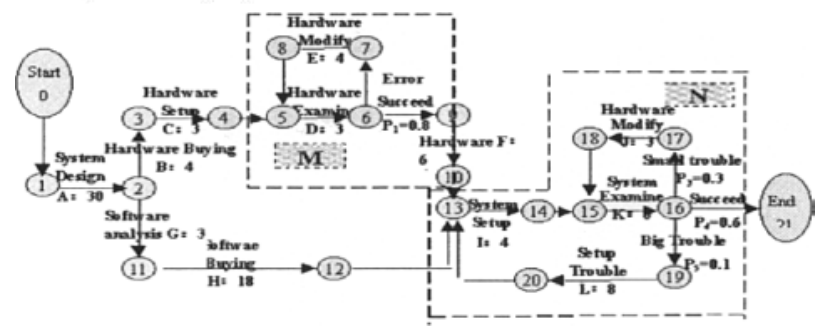

Figure 9. GERT Network of IT Project

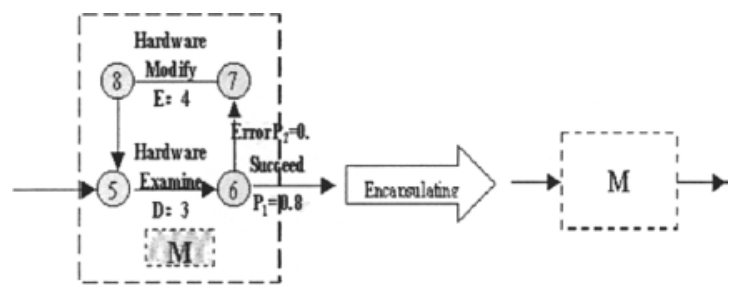

Figure 10. Modified Activity $M$ after Encapsulating

\subsection{Encapsulating Reworking Activities}

In this example, there exist reworking in two activities. They can be encapsulated to two adjusted activities $\mathrm{M}$ and $\mathrm{N}$, as shown in Fig.10 and Fig.11.

\subsection{Computing of Expected Duration of Modified Activity}

(1) Expected Duration of Adjusted Activity M

According to Equation (2), expected duration of adjusted activity $\mathrm{M}$ can be computed as following, 


$$
\mathrm{D}_{\mathrm{M}}=\frac{D_{E}+D_{D}}{P_{1}}=\frac{3+4}{0.8}=8.75
$$

(2) Expected Duration of Modified Activity N

Because activity $\mathrm{K}$ exist three path choices, according to Equation (10), expected duration of adjusted activity $\mathrm{N}$ can be computed as following,

$$
\begin{gathered}
\mathrm{D}_{16,21}=\frac{\mathrm{P} 3 * 9+\mathrm{P} 5 * 18}{0.6}=\frac{4.5}{0.6}=7.5 \\
\mathrm{D}_{13,21}=\mathrm{D}_{13,14}+\mathrm{D}_{14,15}+\mathrm{D}_{15,16}+\mathrm{D}_{16,21}=4+0+6+11.25=21.25
\end{gathered}
$$

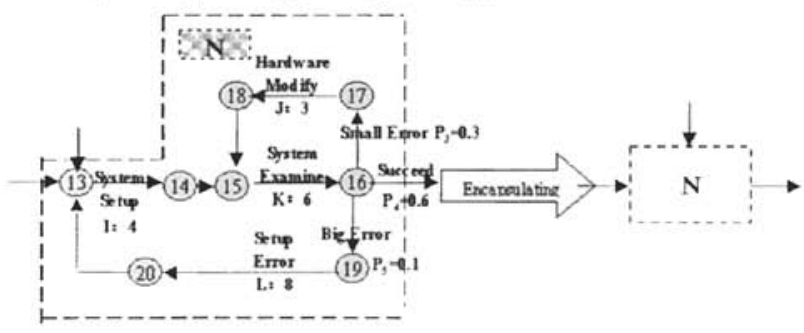

Figure 11. Modified Activity $\mathrm{N}$ after Encapsulating

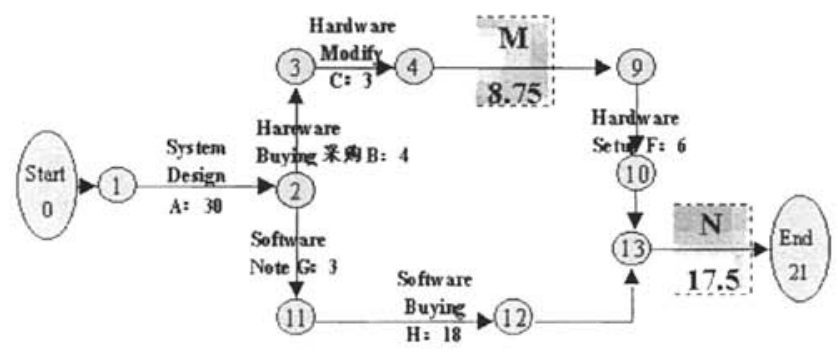

Figure 12. Modified GERT Network Without Reworking

\subsection{Generating Adjusted GERT Network}

After encapsulating all reworking activities and computing their adjusted duration, we can get adjusted GERT network without reworking, as shown in Fig.12.

\subsection{Computing of Expected Critical Path and Expected Duration}

Using CPM techniques, we can get expected project critical path,

$$
\mathrm{A}-\mathrm{B}-\mathrm{C}-\mathrm{M}-\mathrm{F}-\mathrm{N}
$$

Then, its expected duration can be computed, 
$\mathrm{D}=30+4+3+8.75+6+21.25=73$ (days)

\section{CONCLUSIONS}

Reworking exists widely in IT project management. The authors studied estimating method on project duration in different modes based on GERT network diagram, and got the general steps of computing project duration. The method not only adapts to GERT network with reworking, also adapts to traditional determinate project network without reworking. Maturity of the method needs to be more verified in the future.

\section{ACKNOWLEDGEMENTS}

This research was supported by Open Foundation of Key Laboratory of Data Engineering and Knowledge Engineering, Ministry of Education P. R. China, Open Foundation of Key Laboratory of Information Management and Economy, Ministry of Information Industry, P. R. China under grants F0607-31, F0607-42, and Research Foundation of Renmin Univ. of China.

\section{REFERENCES}

1. K. Kenzo and N. Nishiuchi, Efficient Monte Carlo simulation method of GERT-type network for project management, Computers and industrial engineering. Volume 42 , Number 2-4, pp.521-531, (2002).

2. E.H. John, Applications of simulation in project management, in Proceedings of the 11th conference on Winter simulation (San Diego, California, United States, 1979), pp.211-219.

3. K. Kim and J.M.D.L. Garza,. Evaluation of the resource-constrained critical path method algorithms, Journal of construction engineering and management-Asce. Volume 131, Number 5, pp.522-532, (2005).

4. A. Azaron, H. Katagiri, and M. Sakawa, A multi-objective resource allocation problem in PERT networks, European Journal of Operational Research. Volume 172, Number 3, pp.838-854, (2006).

5. C.H. Cheng, Fuzzy repairable reliability-based on fuzzy GERT, Microelectronics and Reliability. Volume 36, pp.1557-1563, (1996).

6. E.R. Clayton and L.J. Moore, GERT vs. PERT, The Journal of System Management. Volume 22, pp.11-19, (1972).

7. E.D. David, PERT and simulation, in Proc. of the 10th conference on Winter simulation (Miami Beach, FL, 1978), pp.89-98. 